\title{
Fault Diagnosis of Axial Piston Pump Based on Extreme-Point Symmetric Mode Decomposition and Random Forests
}

\author{
Lei Yafei $\mathbb{D}^{1},{ }^{1}$ Jiang Wanlu $\mathbb{D}^{2},{ }^{2}$ Niu Hongjie $\mathbb{D}{ }^{3}$ Shi Xiaodong $\mathbb{D}{ }^{2}$ and Yang Xukang $\mathbb{D}^{2}$ \\ ${ }^{1}$ School of Mechanical and Electrical Engineering, North China Institute of Aerospace Engineering, Langfang 065000, China \\ ${ }^{2}$ College of Mechanical Engineering, Yanshan University, Qinhuangdao 066004, China \\ ${ }^{3}$ School of Computer and Remote Sensing Information Technology, North China Institute of Aerospace Engineering, \\ Langfang 065000, China \\ Correspondence should be addressed to Jiang Wanlu; wljiang@ysu.edu.cn
}

Received 3 December 2020; Revised 3 March 2021; Accepted 29 March 2021; Published 2 July 2021

Academic Editor: Moon G. Lee

Copyright $\odot 2021$ Lei Yafei et al. This is an open access article distributed under the Creative Commons Attribution License, which permits unrestricted use, distribution, and reproduction in any medium, provided the original work is properly cited.

\begin{abstract}
Aiming at fault diagnosis of axial piston pumps, a new fusion method based on the extreme-point symmetric mode decomposition method (ESMD) and random forests (RFs) was proposed. Firstly, the vibration signal of the axial piston pump was decomposed by ESMD to get several intrinsic mode functions (IMFs) and an adaptive global mean curve (AGMC) on the local side. Secondly, the total energy was selected as the data of feature extraction by analyzing the whole oscillation intensity of the signal. Thirdly, the data were preprocessed and the labels were set, and then, they were adopted as the training and testing set of machine learning samples. Lastly, the RFs model was created based on machine learning service (MLS) to diagnose the faults of the axial piston pump on the cloud. Using the test and verifying the data set for comparative testing, the fault diagnosis precision rates of the model are above $90.6 \%$, the recall rates are more than $90.9 \%$, the F1 score is higher than $90.7 \%$, and the accuracy rate of this model reached $97.14 \%$. A benchmark data simulation of mechanical transmission systems and an experimental data investigation of an axial piston pump are performed to manifest the superiority of the present method by comparing with classification and regression trees (CART) and support vector machine (SVM).
\end{abstract}

\section{Introduction}

The piston pump is a positive displacement pump that can be classified into three main categories, namely bend axis piston pump, radial piston pump, and swash plate axial piston pump. In the paper, the pump in consideration is a swash plate axial piston pump, often called axial piston pump. It is widely used in metallurgical machinery, aerospace, engineering equipment fields, and so forth [1-2]. As the power component of the hydraulic system, it determines the pressure and flow of the whole system. The failure of the axial piston pump will cause huge economic losses and even serious safety accidents. Moreover, axial piston pump usually worked continuously under high pressure and adverse working environment, and the contaminated oil is easy to cause its failure. It is necessary to study the fault diagnosis of the axial piston pump [3-6].
In academic research and practical applications, many studies have been conducted on the widely used faulty feature extraction, fault diagnosis algorithm fusion, and intelligent diagnosis applications [7-10]. In terms of extraction of fault features, Wang et al. applied the minimum entropy deconvolution, Walsh transform denoising, Teager energy operator (TEO) demodulation, and other methods to the fault diagnosis of axial piston pump, which effectively solved the technical problem of extracting fault features from vibration signals contaminated by strong background noise [11-13]. Casoli et al. presented a feature extraction using principal components analysis (PCA) techniques and different classification to detect the health state of a variable displacement axial-piston pump based on vibration signals [14]. Li et al. proposed a fault feature selection method for axial piston pump based on quantum genetic algorithm [15]. Liu et al. employed a new method for assessing the status of 
multi-information intensity features based on fault mechanism for the wear fault of hydraulic pump slipper [16]. Gao et al. applied TEO demodulation using improved clusteringbased segmentation and L-Kurtosis method to extract fault features in axial piston pumps. Wang et al. employed a sparse coding method to extract weak features of the failure hydraulic pump [17]. Buono et al. applied a diagnosis method of accelerometer time series analysis based on autoregressive and moving average (ARMA) models which has been used to figure out the pump failure [18].

To integrate multiple fault diagnosis algorithms, Tang et al. proposed a fault diagnosis method based on empirical mode decomposition (EMD) and envelope spectrum aiming at the difficulty in early fault diagnosis of the pump. Jia et al. proposed a novel fault diagnosis method based on symbolic perceptually important point (SPIP) and hidden Markov model (HMM) [19]. Liu et al. proposed a loose-slip fault diagnosis method for axial piston pumps based on minimum entropy deconvolution, ensemble empirical mode decomposition, and extreme learning machine [20]. Jiang et al. integrated local mean decomposition and improved adaptive multiscale morphology analysis for hydraulic pump fault signals demodulation. Lu et al. proposed a fault diagnosis method combining a two-step EMD method based on waveform matching and extrema mirror extension with fuzzy C-means clustering to identify the faults of pumps [21]. Tian et al. applied a fault diagnosis method for hydraulic pumps based on local mean decomposition (LMD), singular value decomposition (SVD), and informationgeometric support vector machine (IG-SVM) [22]. Zheng et al. proposed a new fusion method based on symplectic geometry mode decomposition (SGMD) and general morphological fractal dimensions (GMFDs) for the fault diagnosis of the hydraulic pump [23].

Aiming at the application of artificial intelligence algorithms, Du et al. combined sensitivity analysis and probability neural network (PNN) to improve the speed and accuracy of the hydraulic pump fault diagnosis [24]. Babikir et al. integrated an artificial neural network (ANN) algorithm, cat swarm optimization (CSO) algorithm, and firefly algorithm (FA) to predict the noise of a submersible axial piston pump [25]. Lv et al. applied local characteristic-scale decomposition (LCD), fuzzy entropy, and self-organization map (SOM) neural network in fault diagnosis of the hydraulic pump [26]. Lázaro and Pinilla proposed a methodology based on Wavelet Analysis and ANN's for the detection of wear in hydraulic axial piston pumps [27]. Shen et al. employed a fault diagnosis method based on wavelet packet analysis and PSO optimizing neural networks [28]. Bensaad et al. presented a new approach based on the physical model of the pump to identify the worn piston from the healthy ones [29]. Cai et al. proposed a fault diagnosis method based on wavelet packet decomposition and directed acyclic graph support vector machines (MDAG SVM) to solve the problems that the fuzzy fault feature of piston pumps have and are difficult to locate accurately [30]. Han et al. also proposed a comprehensive evaluation method of variational mode decomposition (VMD) and support vector data description (SVDD) to evaluate the performance degradation of hydraulic pumps quantitatively [31]. To summarize, the above diagnostic methods are highly dependent on experience and people, which possess some limitations in practical applications.

Compared with traditional methods, ESMD can not only smooth a series of complex signals, but also effectively solve the mode superposition problem in EMD. In addition, the total energy of the pulsating mode can be calculated on the basis of ESMD modal decomposition, and the energy variation characteristics can be used in the fault diagnosis process [32-35]. And RFs is an integrated algorithm, and, compared with CART, there is no need to prune a large number of sample data, and it is not easy to overfit. It is insensitive to outliers and noise data, can maintain high accuracy, can process high-dimensional data, and has the advantages of parallel processing ability and scalability. It can be applied to various environments such as clustering analysis and fault diagnosis classification [36-39]. Therefore, the advantages of ESMD and RFS are combined and applied to the research of axial piston pump fault diagnosis.

As we all know, it is the data-driven era nowadays. It is an important tendency to the fault diagnosis of axial piston pump based on software as a service (SaaS). MLS is a new data mining and analysis platform. It integrates multiple algorithm nodes to create a visual workflow based on a research task for data processing, model training, evaluation, and prediction [40-41]. However, the fault diagnosis of axial piston pumps, data with higher acquisition frequency, is often required. It is necessary to perform feature extraction by edge computing to reduce the data dimension and facilitate the transmission of information to the cloud platform [42, 43]. In this paper, ESMD is used to extract the fault characteristics of the condition monitoring signal of the axial piston pump on the edge side, and RFs are used to construct the diagnostic model in MLS.

The outline of the paper is as follows: Section 2 summarizes the principle of ESMD and RFs. In Section 3, simulation verification is conducted with the benchmark data. In Section 4, the axial piston pump fault simulation test bench is introduced. In Section 5, the proposed method is applied to analyze the vibration signals from an axial piston pump. Section 6 concludes the proposed approach and shows future work regarding data analytics.

\section{A Brief Review of ESMD and RFs}

2.1. The Principles of ESMD. The ESMD usually adopts oddeven pole symmetric modal decomposition, and the algorithm is as follows [32]:

Step 1: find all the extreme points (maximum and minimum) of the original signal $Z$ and mark them as $E_{i}$ $(i=1,2, \ldots, n)$.

Step 2: connect all adjacent extremum points $E_{i}$ with a line segment, and mark the midpoint of the line segment as $O_{i}(i=1,2, \ldots, n-1)$.

Step 3: the middle points $\mathrm{O} 0$ and $\mathrm{On}$ of the left and right boundary are supplemented by linear interpolation. 
Step 4: two interpolation curves L1 and L2 were constructed based on the Ordinal Numbers of odd and even numbers of the obtained $n+1$ midpoints. Then L1 is generated by interpolation of the midpoint of odd ordinals, L2 is generated by interpolation of the midpoint of even ordinals, and their mean curve $\mathrm{L}^{*}$ is calculated:

$$
L^{*}=\frac{L_{1}+L_{2}}{2}
$$

Step 5: for data $Z-L^{*}$ after removing the mean curve $L^{*}$, repeat steps 1-4 until ( $\varepsilon$ is the set allowable error) the filter times reach the preset value $S$. Now, we have our first mode $M_{1}$.

Step 6: repeat the above steps $1-5$ for $Z-M_{1}$ to obtain $M_{2}, M_{3}, \ldots$ until the residual component $r$ meets the termination condition: the number of extreme points in $r$ does not exceed the preset value.

Step 7: let the filter times $S$ change continuously within the integer interval $\left[S_{\min }, S_{\max }\right]$, and repeat the above steps 1-6 to obtain a series of decomposition results. Then, calculate the variance $\sigma_{1}^{2}$ of data Z- $r$ after removing the residual $r$ and the standard deviation $\sigma_{0}$ of data $Z$, and draw the relation curve of mean-variance ratio $\sigma_{1} / \sigma_{0}$ with respect to $S$.

$$
\begin{aligned}
\sigma_{1}^{2} & =\frac{1}{N} \sum_{i=1}^{N}\left(z_{i}-r_{i}\right)^{2}, \\
\sigma_{0}^{2} & =\frac{1}{N} \sum_{i=1}^{N}\left(z_{i}-\bar{Z}\right)^{2}, \\
\bar{Z} & =\frac{1}{N} \sum_{i=1}^{N} z_{i},
\end{aligned}
$$

where $z_{i}$ is the sample value of data $Z ; r_{i}$ is the sample value of the margin $r ; \bar{Z}$ is the global mean value of the data $Z ; N$ is the number of original data sampling points.

Step 8: find the value $S_{0}$ corresponding to the minimum mean square error ratio $\sigma_{1} / \sigma_{0}$ in the integer interval $\left[S_{\min }, S_{\max }\right]$; set $S=S_{0}$, and repeat the above steps 1-6 to regain all modes $M_{1}, M_{2}, \ldots$ and the margin $r$. At this point, the resulting margin $r$ is an optimal AGM curve.

It can be seen from the above steps that the ESMD method can decompose the signal into some intrinsic mode functions (IMFs) and a residual component $r$. In practical application, it is usually selected as $\varepsilon=0.001 \sigma_{0}$. And the mean-variance ratio $\sigma_{1} / \sigma_{0}$ is used to represent the optimization degree of the mean $\bar{Z}$ for the margin $r$.

Since the amplitude of the pulsation mode reflects the amount of energy, and the amplitude of each pulsation mode varies with time, so the energy variation characteristics can be taken as the research object.

After the original signal is decomposed by ESMD, a series of eigenmode functions will be obtained. Among them, the analytic expression of the $j$-th eigenmode function can be expressed as

$$
x_{j}(t)=A_{j}(t) \cos \theta_{j}(t), \quad(1 \leq j \leq n) .
$$

Among them, $A_{j}(t)$ is the $j$-th eigenmode amplitude function; $\theta_{j}(t)$ is the $j$-th eigenmode phase function.

In the form of odd-even pole symmetric mode decomposition, the amplitude function $A_{j}(t)$ has the characteristic of slow variation. The total energy can be defined as the form of wave energy:

$$
E(t)=\frac{1}{2} \sum_{j=1}^{n} A_{j}^{2}(t) .
$$

For the vibration acceleration signal, the total energy is represented as the overall oscillation intensity of the vibration pulsation.

2.2. Algorithm Principle of RFs. RFs is an integrated learning algorithm based on a decision tree classifier. Combining the advantages of the Bagging algorithm and random subspace algorithm, it introduces two randomization processes so that each tree has different classification capabilities. Integrated learning has better classification performance than a single classifier and can effectively $\left\{h\left(X, \theta_{k}\right), k=1,2, \ldots, K\right\}$ improve the generalization ability of the learning system.

2.2.1. Principle of RFs. RFs is a combinatorial classifier based on the $K$ decision tree $\theta_{k}$, where $K$ is a sequence of independent and identically distributed random variables; $X$ is the input variable of the classifier. Basic principle: first, the Bootstrap resampling method was used to randomly extract $K$ sample training sets from the training set, and the size of each sample training set was consistent with that of the training set; then $K$ decision tree models were established for $K$ sample training sets, and $K$ classification results were obtained. Finally, vote on each result according to $K$ classification results to determine the optimal classification [44]. The specific process is shown in Figure 1.

After $\left\{h_{k}(X), k=1,2, \ldots, K\right\}$ times of training, a classification model sequence will be obtained, from which a multiclassification model system will be formed. The final classification result of the system adopts the simple majority voting method, and the classification decision expression is as follows:

$$
H(X)=\arg \max _{Y} \sum_{k=1}^{K} I\left(h_{k}(X)=Y\right),
$$

where $H(X)$ represents the combined classification model; $h_{k}(X)$ is a single classification model; $Y$ stands for the correct category; $I(\cdot)$ is the Indicator function.

2.2.2. RFs Convergence. Set a group of the classification model $\left\{h_{k}(X), k=1,2, \ldots, K\right\}$, and the sample training set of each classification model is randomly sampled from training set $X$; then, the remaining Margin function is 


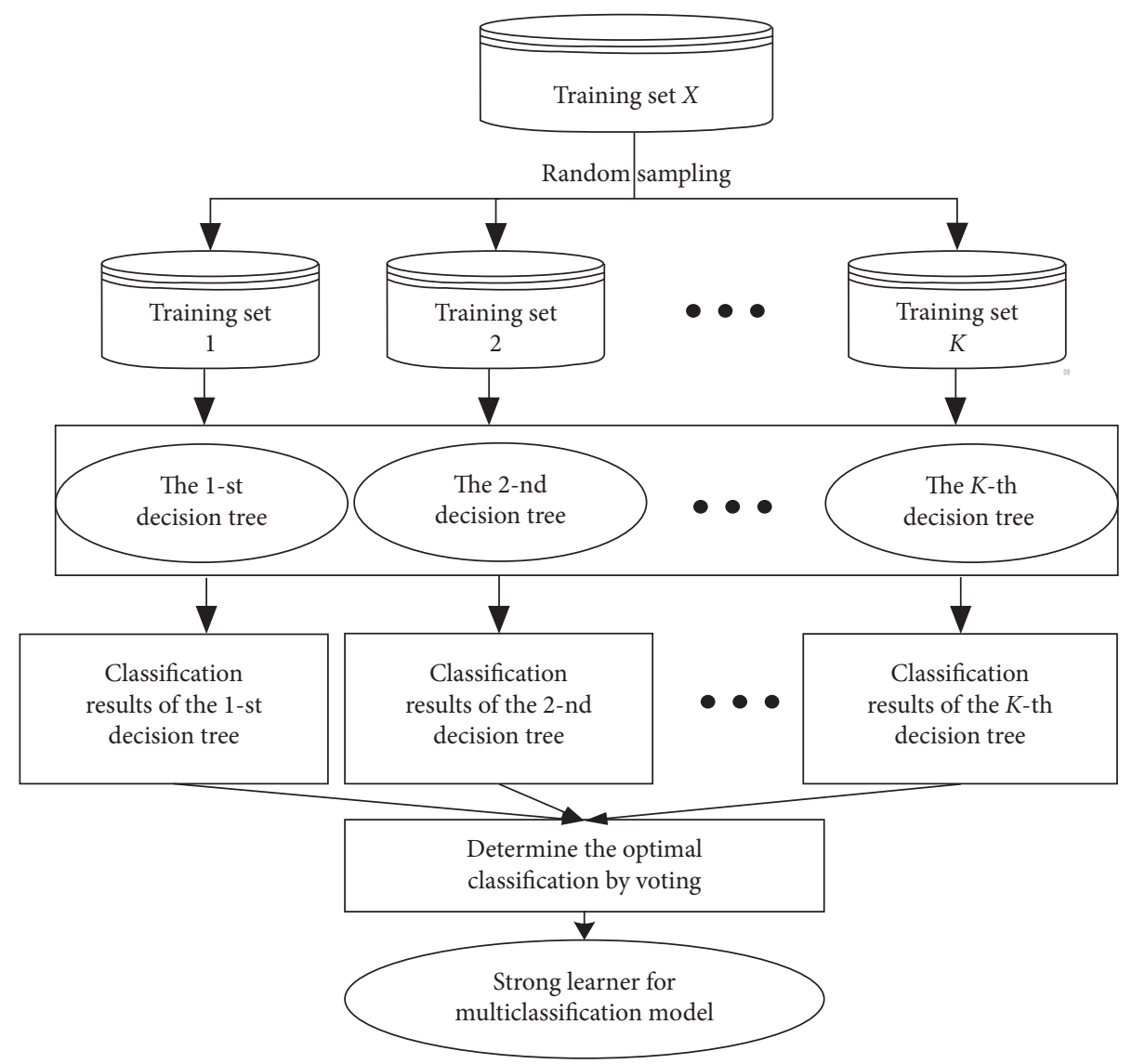

FIGURE 1: Flow chart of RFs algorithm.

$$
m g(X, Y)=a v_{k} I\left(h_{k}(X)=Y\right)-\max _{j \neq Y} a v_{k} I\left(h_{k}(X)=j\right),
$$

where $I(\cdot)$ is the Indicator function; $Y$ is the correct category; $j$ is the incorrect category; and $a v_{k}(\cdot)$ is the average.

The margin function is the degree to which the number of votes in the correct category exceeds the number of votes in the incorrect category. The larger the margin function, the higher the confidence of the classifier. Generalization error is deduced as follows:

$$
P E^{*}=P_{X, Y}(m g(X, Y)<0) .
$$

In RFs, $h_{k}(X)=h\left(X, \theta_{k}\right)$, and when the decision tree classification model is large enough, it obeys the law of large numbers. With the increase of the classification model of a decision tree, it can be proved that the generalization error $P E^{*}$ of all random sequences $\left\{\theta_{k} n k=1,2, \ldots, K\right\}$ converges to the following expression:

$$
P_{X, Y}\left(P_{\theta}(h(X, \theta)=Y)-\max _{j \neq Y} P_{\theta}(h(X, \theta)=j)<0\right) .
$$

It can be seen from (8) that RFs does not cause the problem of overfitting with the increase of the decision tree and has convergence.
2.2.3. Quantitative Evaluation of Tree Nodes. RFs is composed of multiple decision trees, each of which is independent of the other. In the decision tree, Entropy or Gini coefficient is often used to quantify the order degree of a set and to quantitatively evaluate the splitting attribute of each node.

(1) Information Entropy. Information entropy is a basic concept in information theory, which is used to represent the disorder degree of a set. The higher the entropy is, the more chaotic the set is or otherwise the more ordered the set is. It is defined as follows: suppose $D$ is a discrete random variable and $\bar{d}_{i}$ is the category of class $i$, then its probability distribution is

$$
P\left(D=\overline{d_{i}}\right)=p_{i}, \quad i=1,2, \ldots, n .
$$

Then, the entropy of the random variable $D$ is

$$
H(D)=-\sum_{i=1}^{n} p_{i} \log _{2} p_{i}
$$

where $p_{i}$ is the proportion of sample size of category $i$ in all samples; $n$ is the number of data categories.

(2) Gini Coefficient. The CART algorithm selects test attributes according to Gini coefficient. This algorithm calculates Gini coefficient for each sample set division. The smaller 
Gini coefficient, the more reasonable the division. It is defined as follows.

In the classification problem, if there are $n$ classes and the probability of sample points belonging to class $i$ is $p_{i}$, Gini coefficient is defined as

$$
\operatorname{Gini}(D)=\sum_{i=1}^{n} p_{i}\left(1-p_{i}\right)=1-\sum_{i=1}^{n} p_{i}^{2}
$$

where $D$ represents all samples of the data set; $p_{i}$ is the proportion of sample size of category $i$ in all samples; $n$ is the number of data categories.

\section{Fault Diagnosis Method and Simulation Verification}

3.1. Fault Diagnosis Using the ESMD and RFs. In this paper, the ESMD method is combined with RFs integrated learning and applied to hydraulic pump fault diagnosis. Firstly, the state monitoring signals of the hydraulic pump are collected, and the effective original signals are selected for fault diagnosis; secondly, the mode decomposition of the selected original signals is carried out by using the ESMD method. Thirdly, the total energy of the signal is calculated as the data source of machine learning feature extraction. Then, the sliding window method is used to calculate the mean, variance, maximum, and minimum value in the window to generate the data set with fault statistical characteristics. Finally, according to the fault status of the hydraulic pump, the machine learning samples are constructed, and the training set and test set are divided according to the proportion. The RFs model is built in MLS and tested and evaluated on cloud platform. The specific method flow is shown in Figure 2.

3.2. Simulation Verification with Benchmark Data. The simulation verification is conducted with the benchmark data from Case Western Reserve University (CWRU) bearing data center. The bearing test rig is shown in Figure 3.

The experimental bearing test consists of a motor, a torque transducer/encoder, and a dynamometer; it provides experimental data of normal and fault bearings. The data we use is generated by drive-end bearings (Type: SKF6205) with a rotational speed of about $1797 \mathrm{rpm}$, and the sampling frequency is set to $12 \mathrm{kHz}$. The experimental data include a normal baseline state and three kinds of fault state of ball, inner race, and outer race fault (at 6 o'clock), which faults were introduced to the test bearings using electrodischarge machining with fault diameters of $0.1778 \mathrm{~mm}$ (Slight fault), $0.3556 \mathrm{~mm}$ (Moderate fault), and $0.5334 \mathrm{~mm}$ (Severe fault). There are 1,000,000 data points in this study, including 10 bearing states, and each state has 100,000 data points.

These data points are built into three simulation validation sample data sets, including slight, moderate, and severe faults state. And each of the simulation validation sample sets contains four kinds of faults: normal state, ball fault, inner race fault, and outer race fault. When we perform simulation verification, ESMD is used to decompose the data sample and obtain the total energy $E(t)$. Then, it is used to solve the mean, variance, maximum, and minimum of total energy $E(t)$ by the sliding window with 20 widths. Finally, the input sample sets to machine learning are constructed, and the sample label setting is shown in Table 1.

In the same environment on the MLS platform, the model constructed by the RFs was compared with the CART model and SVM model. The comparison diagram is displayed in Figure 4.

The feature set based on ESMD is randomly divided into the training set and test set according to the ratio of $7: 3$ on MLS. Then the comparison is made based on the model evaluation indicators by confusion matrices. The comparison results from slight, moderate, and severe fault states are shown in Figure 5.

Figure 5 shows the normalized confusion matrix of the test set after ESMD under different classifiers. Figures 5(a)5(c) are the result of RFs, where each pattern is identified as itself above $92 \%$, the most of which are correctly classified; Figures $5(\mathrm{~b}))-5(\mathrm{~h})$ are the result of CART classifier, where each pattern is identified as itself only more than $58 \%$, in which there are more data judgment errors. Figures 5(g))5(i) show that not only normal state is misjudged as fault but also fault state is misjudged as normal; the most state's data of SVM classifier have been misjudged. The classification results of RFs classifier and CART classifier are similar, and the diagonal value of the confusion matrix is much larger than the value of the nondiagonal line, but RFs classifier is better than CART classifier. Next, the ESMD-RFs method is used for axial piston pump fault diagnosis.

\section{Axial Piston Pump Fault Diagnosis Test Rig}

4.1. Principle of Hydraulic System. The axial piston pump fault test bench adopts a double-pump oil supply system. The axial piston pump supplies oil to the system, while the blade pump is the oil supply of the axial piston pump to prevent its suction. The experimental object is axial piston pump (MCY14-1B). The rated speed of the driving motor (Y132M$4)$ is $1480 \mathrm{rpm}$ and the working pressure is set at $15 \mathrm{MPa}$. The experimental principle is shown in Figure 6.

4.2. Signal Acquisition Sensor Layout. Three piezoelectric vibration acceleration sensors (YD72D) glued to the pump end are responsible for collecting the fault status monitoring signals of the axial piston pump, and the sampling frequency is set as $f_{s}=50 \mathrm{kHz}$, where the installation positions of the vibration acceleration sensor are perpendicular to each other. The horizontal direction is defined as the $x$-axis, the vertical direction is defined as the $y$-axis, and the parallel direction along the pump axis is defined as the $z$-axis, as shown in Figure 7.

4.3. Fault Injection of Axial Piston Pump. In the process of experiment, fault injection is carried out by replacing different types of fault components with normal components, and five fault operation states of axial piston pump, such as normal state, single slipper wear, single loose slipper, swash plate wear, and center spring wear, are reproduced 


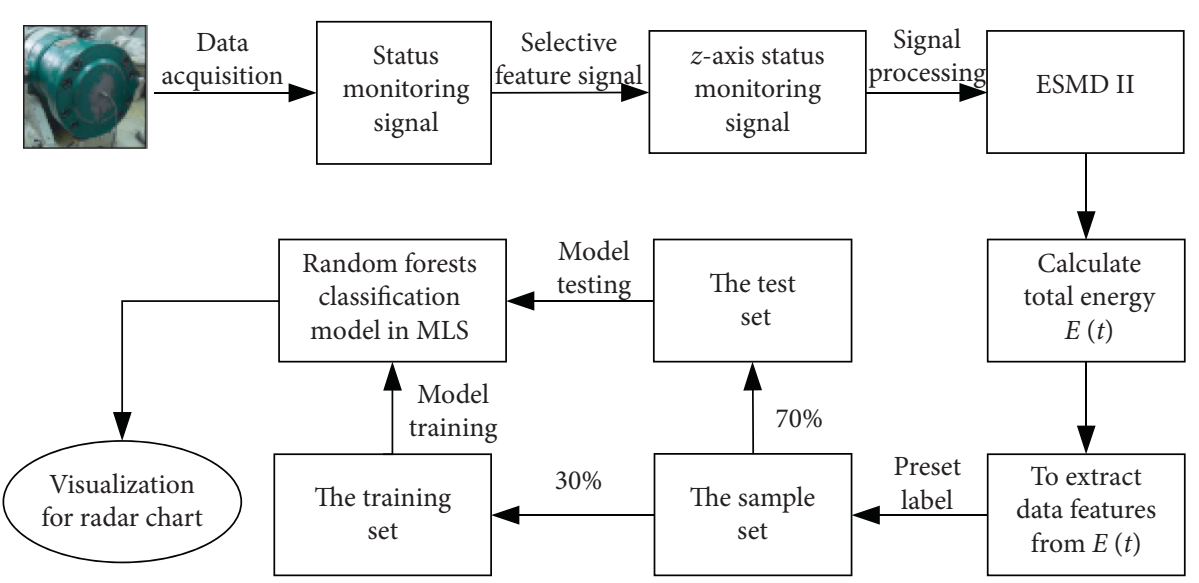

Figure 2: Flow chart of the proposed method.

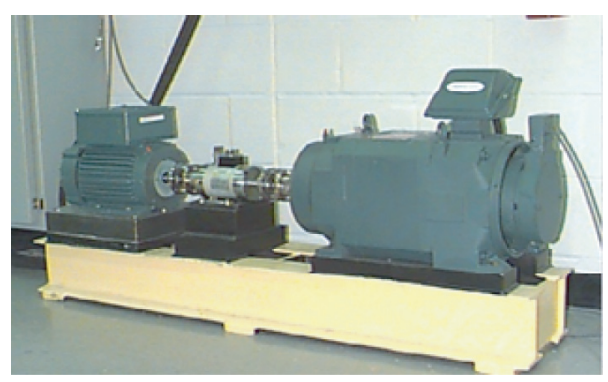

FIGURE 3: CWRU bearing test rig.

TABLe 1: The label of bearing faults.

\begin{tabular}{lcccc}
\hline Bearing status & Normal state & Ball fault & Inner race fault & Outer race fault \\
\hline Label & 0 & 1 & 2 & 3 \\
\hline
\end{tabular}

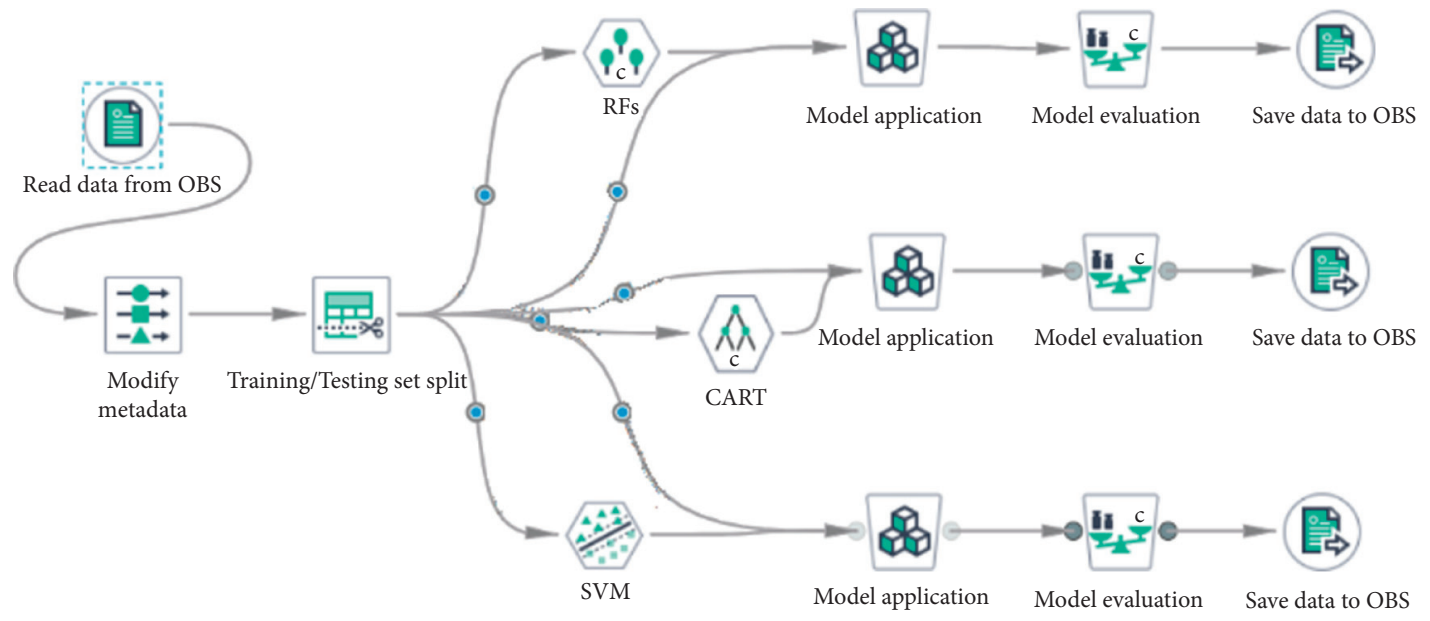

FIGURE 4: Model comparison and simulation analysis.

respectively. These fault components are provided by the hydraulic pump maintenance unit, which can reproduce the running state of the axial piston pump when the actual failure occurs. Among them, the part of fault components is shown in Figure 8.

\section{Fault Diagnosis Process and Built Model}

5.1. Status Monitoring Signal Selection. Since the axial piston pump is rigidly connected with the drive motor by a coupling, it can be known that the pump speed is $n=1480 \mathrm{rpm}$, 


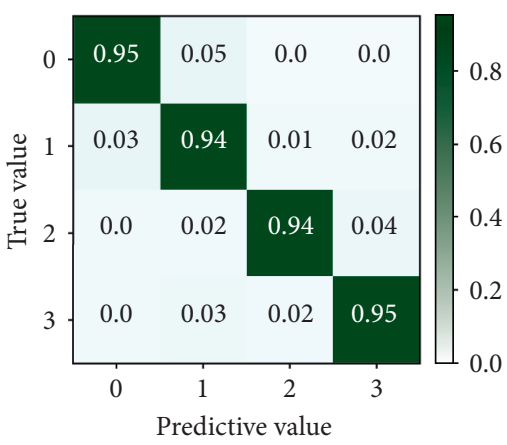

(a)

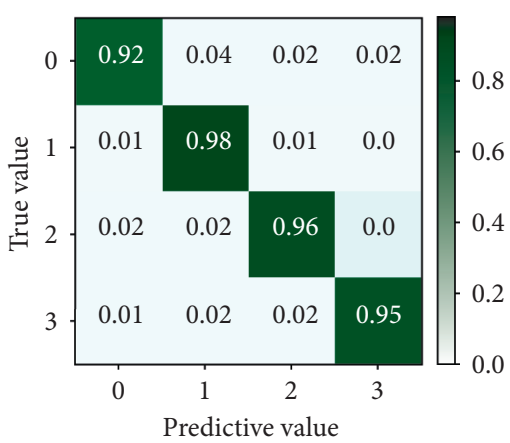

(d)

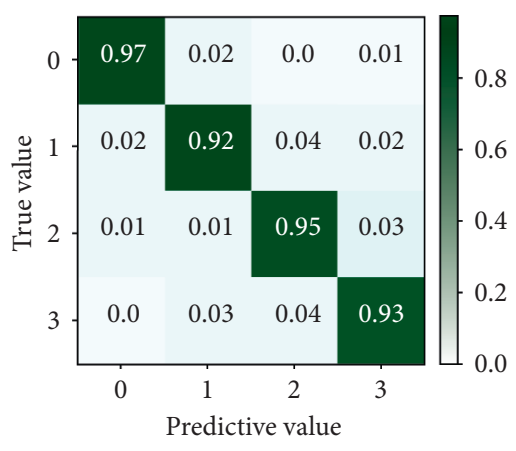

(g)

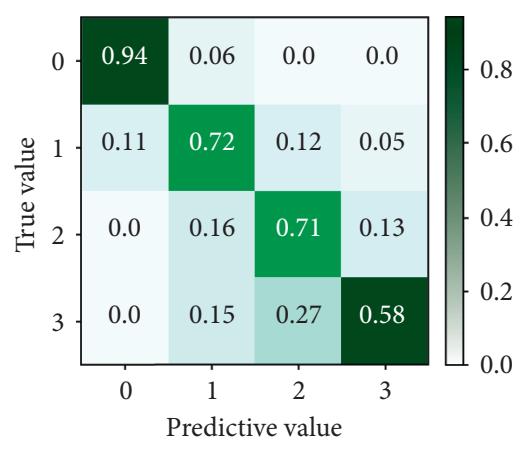

(b)

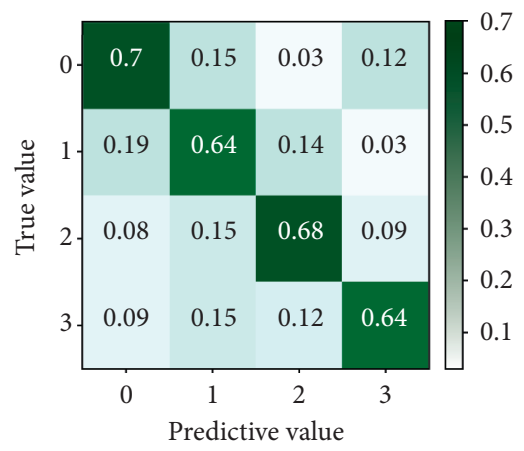

(e)

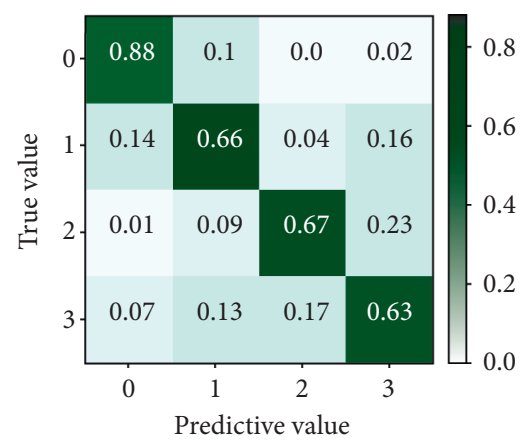

(h)

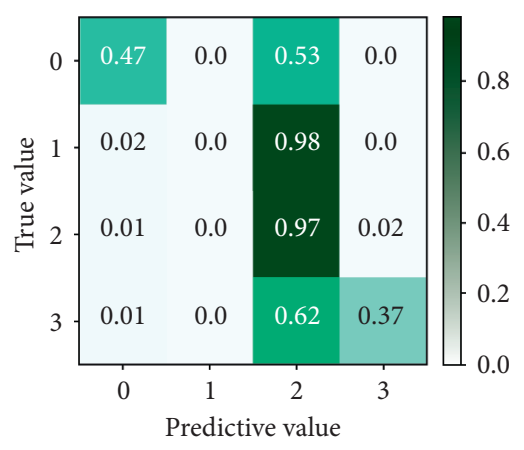

(c)

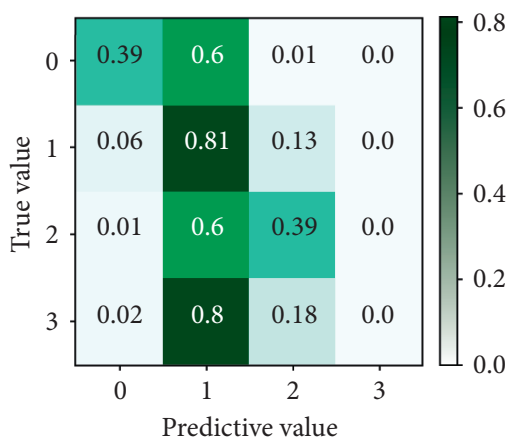

(f)

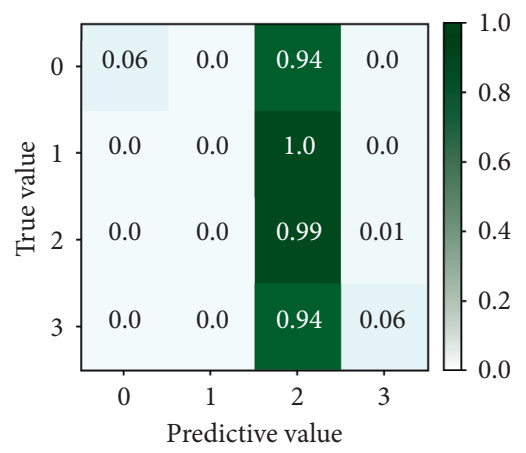

(i)

Figure 5: Multiclass confusion matrices in simulation verification. (a) Slight fault diagnosis based on RFs. (b) Slight fault diagnosis based on CART. (c) Slight fault diagnosis based on SVM. (d) Moderate fault diagnosis based on RFs. (e) Moderate fault diagnosis based on CART. (f) Moderate fault diagnosis based on SVM. (g) Severe fault diagnosis based on RFs. (h) Severe fault diagnosis based on CART. (i) Severe fault diagnosis based on SVM.

and the rotation period $T$ of the pump can be calculated as follows:

$$
T=\frac{60}{n}=\frac{60}{1440} \approx 0.04(s) .
$$

In the collected normal no-fault state signal of axial piston pump, the time-domain waveform signals of vibration acceleration in three directions of 1 cycle (and $0.04 \mathrm{~s}$ ) are randomly intercepted, as shown in Figure 9.

It can be seen from Figure 9 that the vibration amplitude of vibration acceleration signals in the $x$ and $y$ directions does not change significantly, while the vibration amplitude of vibration acceleration in the $z$-direction changes significantly 7 times within 1 period, which accurately reflects the physical characteristics that 7 plungers have one impact on the swash plate in each period. Therefore, the $z$-direction state monitoring acceleration sensor can sensitively pick up the running state change characteristics of the axial piston pump, which can be used as an effective data source for datadriven modeling.

5.2. Original Data Acquisition. Through the observation and analysis of the signal data of the axial piston pump, the original signals of vibration acceleration in the $z$-direction of the collected axial piston pump were studied in five operating states, including normal fault-free, piston slipper wear fault, piston loose shoe wear fault, swash plate wear fault, and center spring wear fault, where the original signal contains 500,000 data points in total and 100,000 data points 


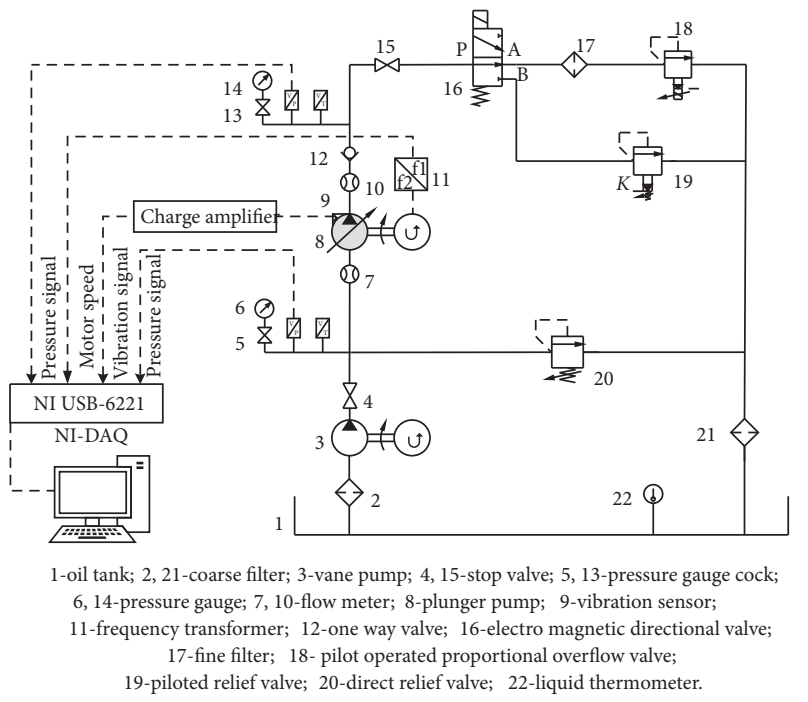

Figure 6: Principle diagram of the test system.

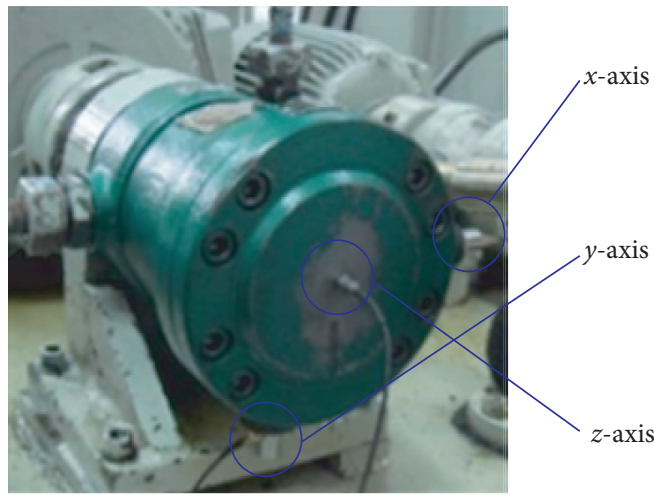

(a)

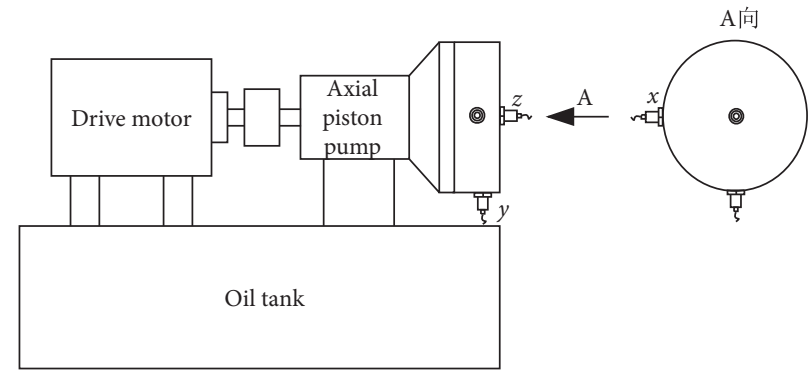

FiguRE 7: Installation of acceleration sensors. (a) Physical map. (b) Sketch map.

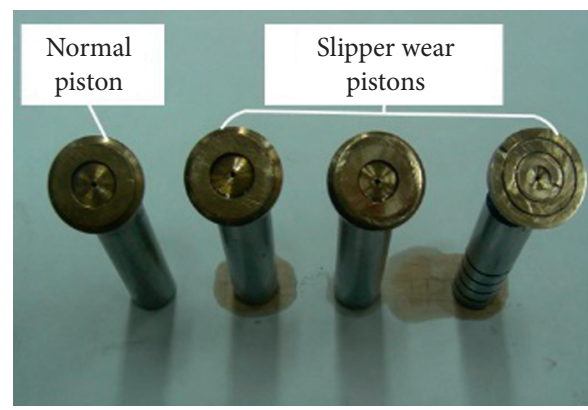

(a)

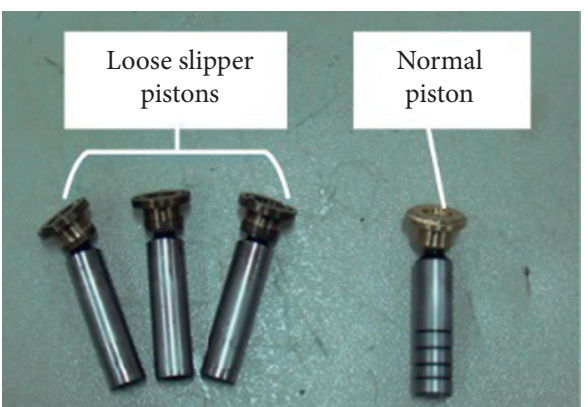

(b)

Figure 8: Continued. 


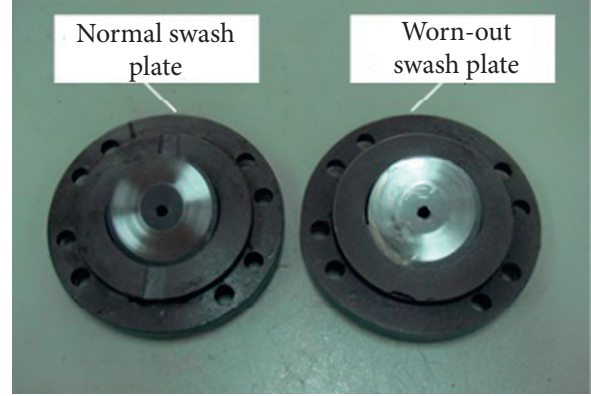

(c)

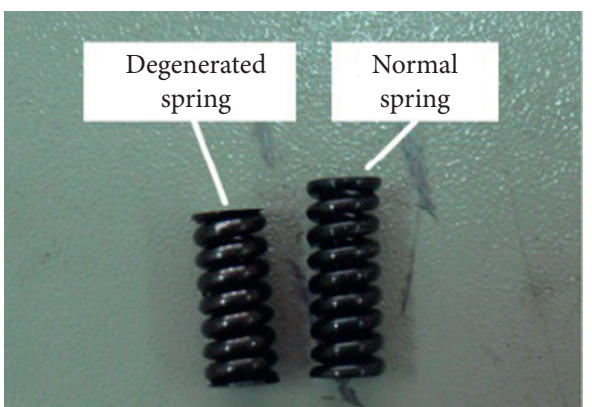

(d)

FIGURE 8: Schematic map of fault injection for axial piston pump. (a) Single slipper wear. (b) Single loose slipper. (c) Swash plate wear. (d) Center spring wear.

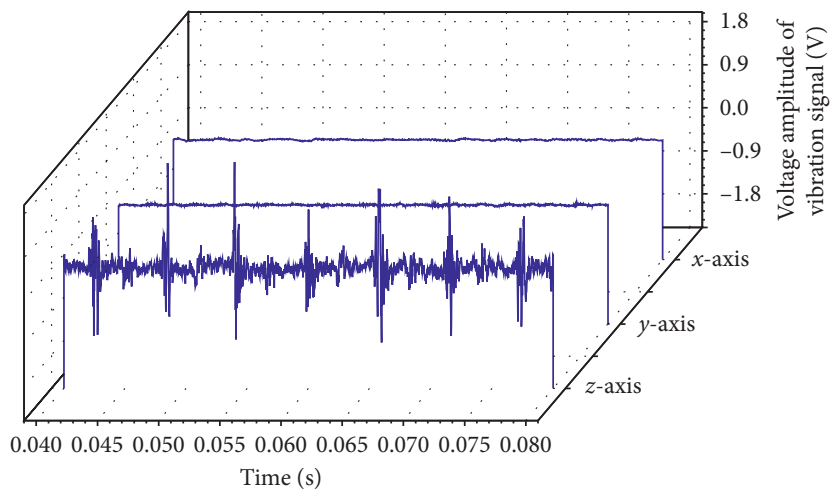

FIGURE 9: The time-domain waveform under the normal state.

in each state and the corresponding sampling time is $2 \mathrm{~s}$. The time-domain waveform of each state is shown in Figure 10.

In addition, according to the requirements of remote cloud machine learning to build training samples and test samples, the original signals are divided in the local edge calculation, where each running state of the axial piston pump was divided into 10 groups, and each data set contained 10,000 data points, corresponding to the $0.2 \mathrm{~s}$ sampling time. Finally, 50 original signal data sets were created.

5.3. Eigenvalue Extraction Based on ESMD. In the process of local edge calculation, the original signal data set is firstly decomposed by ESMD method, and then the eigenvalues of the original signal are extracted by calculating the total energy $E(t)$. The following is an example of the original signal data set of swash plate wear fault of one set of axial piston pumps.

5.3.1. Based on ESMD Mode Decomposition. The ESMD method is used to adaptively decompose the original signal data of swash plate wear fault of axial piston pump, and the corresponding decomposition mode is obtained as shown in Figure 11.

The residual component $r$ obtained by decomposition is an optimized AGMC, which can be considered as the trend term of the corresponding original signal.

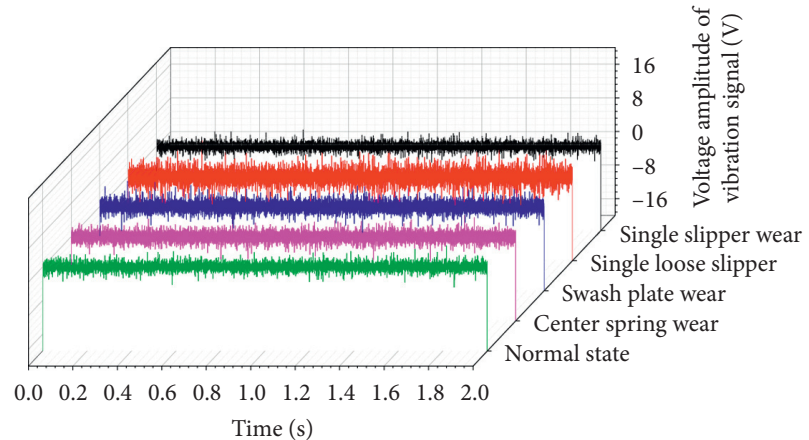

FIgURE 10: The time-domain waveform of vibration signal in $(\mathrm{z})$ axis.

5.3.2. Total Energy of Feature Extraction Value $E(t)$. Under different fault states of axial piston pump, the energy generated by the fault-induced vibration is distinct, and the amplitude of the eigenmode function obtained by ESMD also varies with time. Based on ESMD, according to (4), the total energy $E(t)$ of the original signal in the rotating state of swash plate wear fault can be obtained, as shown in Figure 12.

All 50 sets of original signal data were decomposed based on ESMD mode and the total energy $E(t)$ was calculated, thus obtaining 50 sets of sample data set that extracted the 


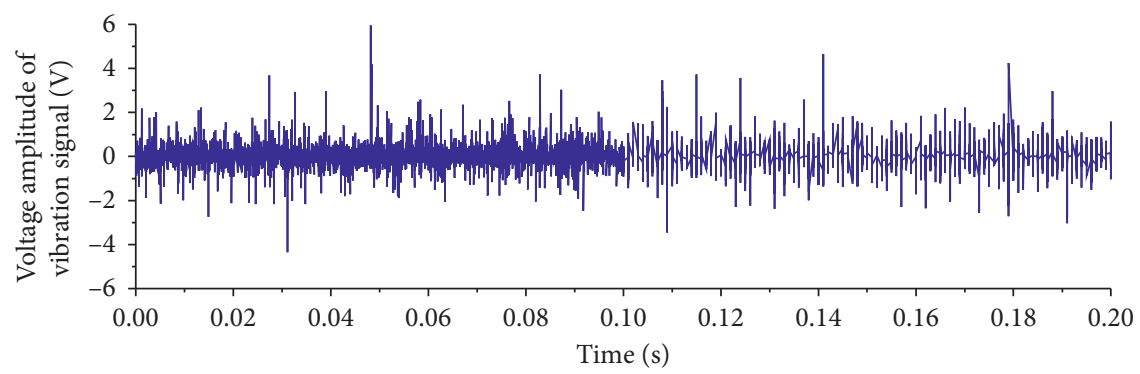

(a)

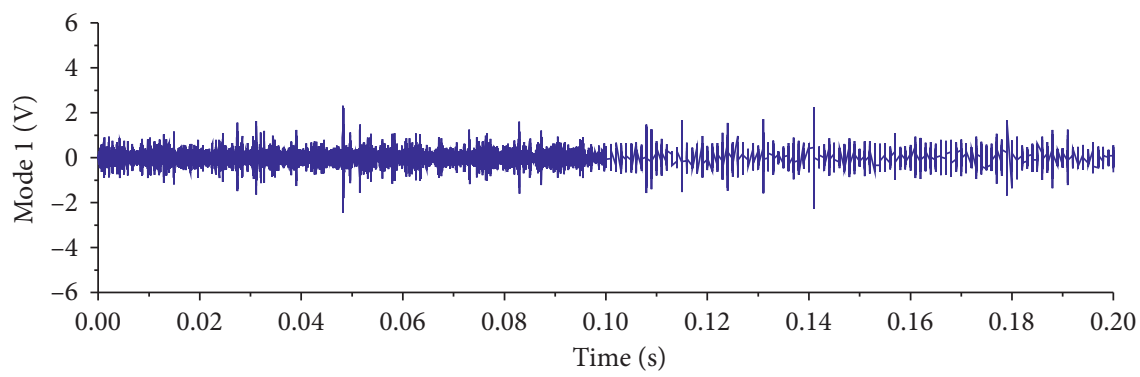

(b)

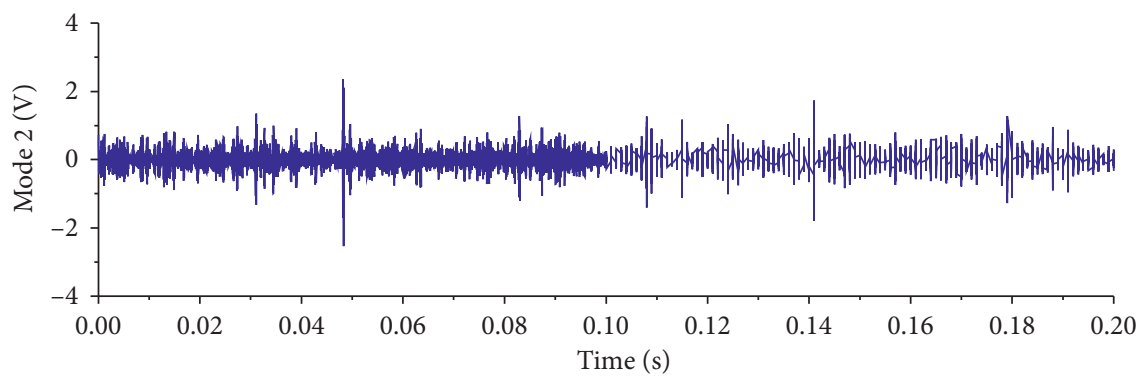

(c)

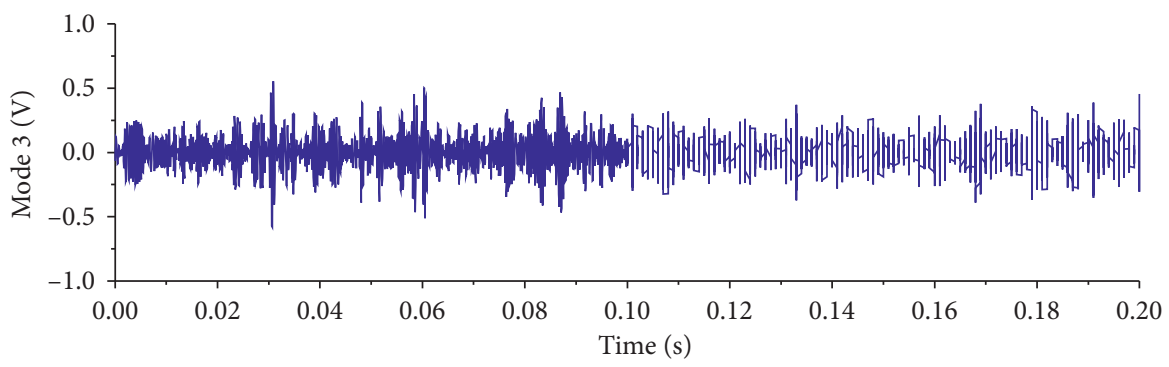

(d)

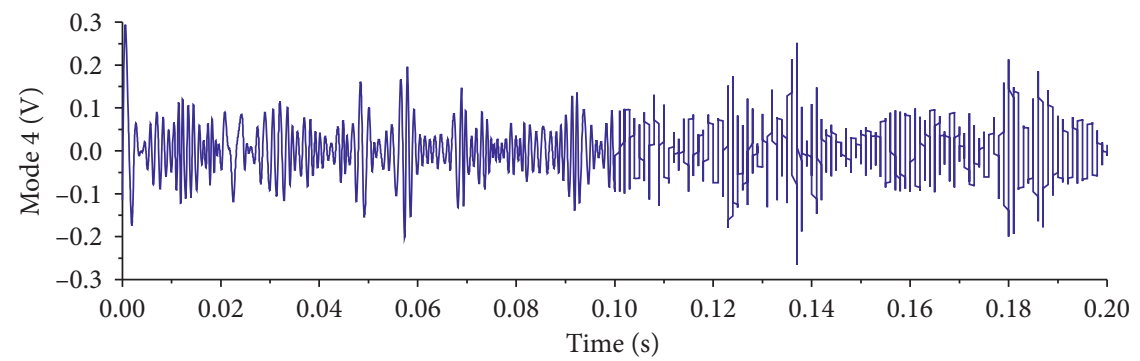

(e)

FIgURE 11: Continued. 


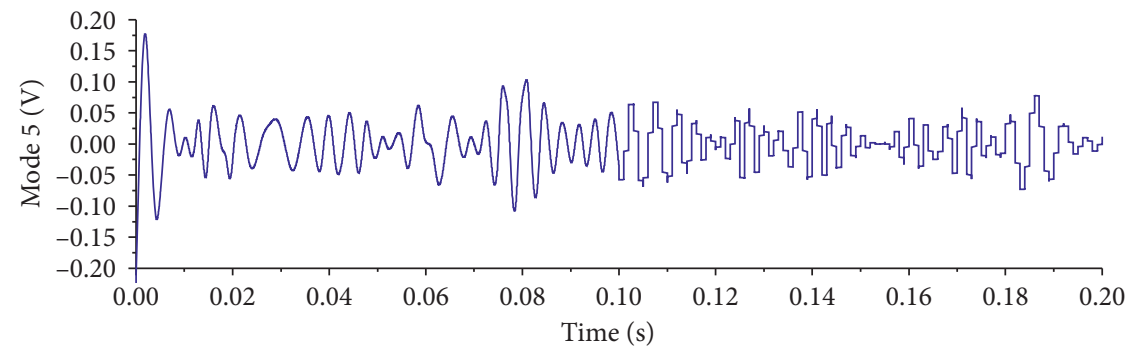

(f)

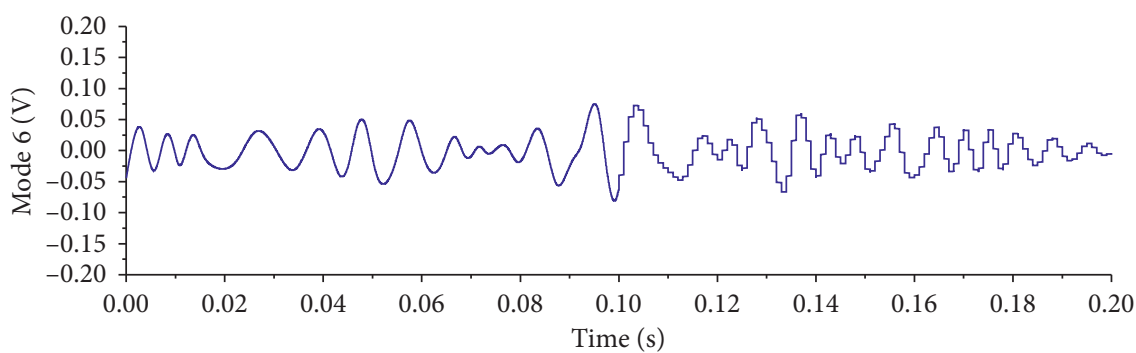

(g)

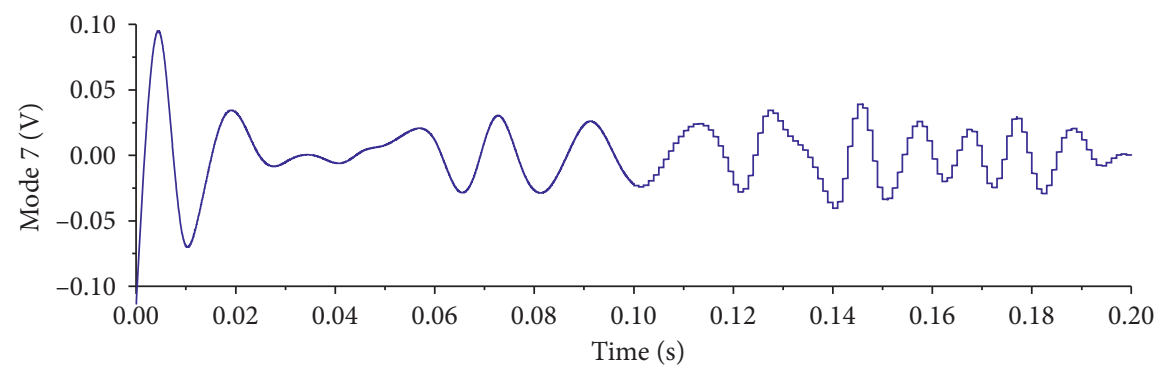

(h)

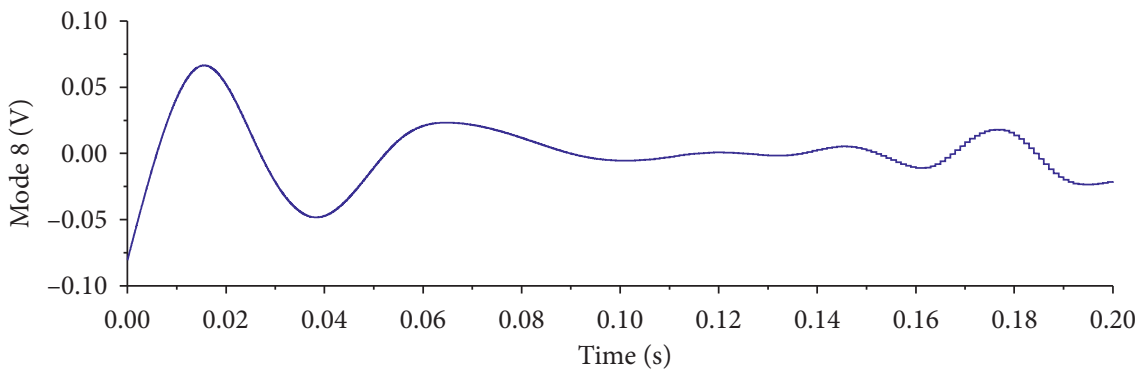

(i)

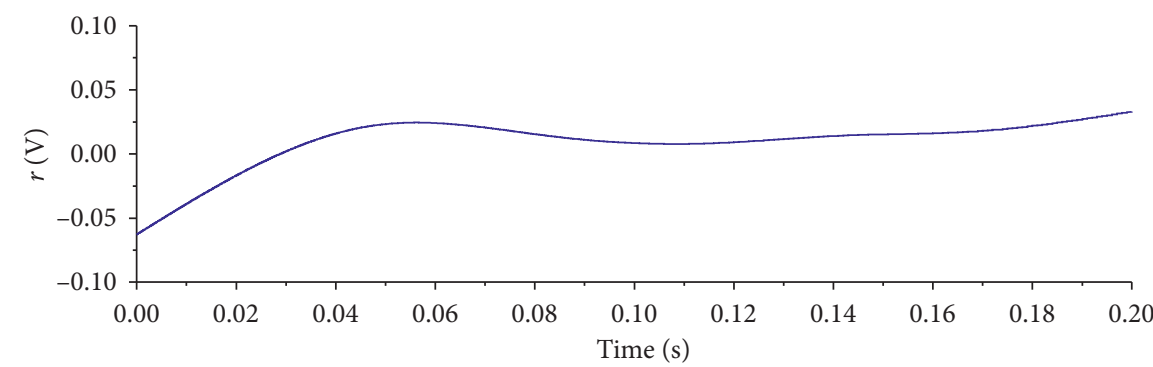

(j)

FigURE 11: Results of ESMD for swash plate wear. 


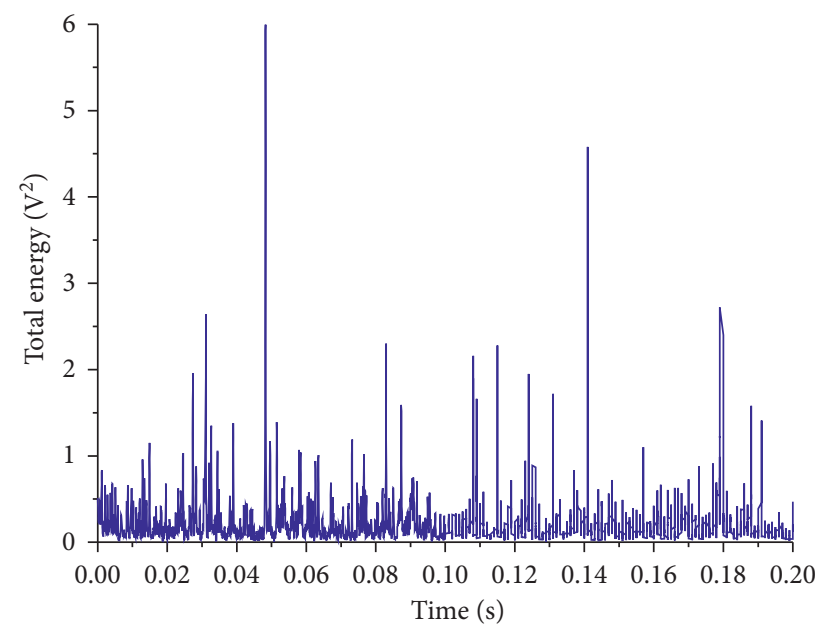

Figure 12: The total energy of swash plate wear for axial piston pump.

characteristic values of different fault states of the axial piston pump.

\subsection{Modeling Based on RFs on MLS}

5.4.1. Build a Machine Learning Sample Set. Cloud machine learning modeling requires high-quality data, and building effective sample sets is an important step and key link in modeling. In this paper, the sliding window method is used to preprocess the data. The specific steps are set a sliding window with a window width of 20 and a step length of 1 in the one-dimensional time series, and calculate the mean, variance, maximum, and minimum statistics of the data in the window, as the input of machine learning samples. Then set the sample label according to the fault state of the axial piston pump (as shown in Table 2), and take the value as the output expectation of the machine learning training sample. Thus, 50 sets of samples suitable for machine learning modeling are constructed.

5.4.2. Model Construction Based on RFs. Considering the balance of machine learning training data, three sets of samples were randomly selected for each fault state of the axial piston pump, a total of 15 sets. Then, the 15 sets of samples were summarized into model training sample data. And then, the workflow of RFs modeling training is constructed in MLS, as shown in Figure 13.

The training model is trained through learning the training samples, adjusting parameters, and optimizing the model in the training process. Finally, the optimal parameters of the RFs model are determined as the number of decision trees in the forest is 200 , the maximum depth of the tree is set to 4 , the decision tree in the forest uses the minimum Gini coefficient to determine the split attribute of internal nodes, and the feature subset selection strategy of RFs selects $\log 2$ and saves it as PMML model file for axial piston pump fault diagnosis.
5.4.3. Model Testing and Evaluation. In the same environment on the MLS platform, the model constructed by the RFs algorithm was compared with the CART classification model and the SVM model based on the axial piston pump fault data again. The comparison is made based on the model evaluation indicators such as the confusion matrix, precision rate, recall rate, and F1-score, which can be seen in Figure 14 .

Figure 14(a) is the result of RFs, pattern 0 is identified as itself with $95 \%$, pattern 1 is identified as itself with $93 \%$, pattern 2 is identified as itself with $94 \%$, pattern 3 is identified as itself with $91 \%$, and pattern 4 is identified as itself with $94 \%$; all of the patterns are identified as themselves more than $91 \%$, the precision rates of the model are above $90.6 \%$, the recall rates are more than $90.9 \%$, and the F1 score is higher than $90.7 \%$. Figure 14 (b) is the result of CART; each pattern is identified as itself only above $60 \%$, and the precision rates of the model are above $60.8 \%$, the recall rates are more than $59.7 \%$, and the F1 score is higher than $60.3 \%$. Figure $14(\mathrm{c})$ is the result of the SVM classifier; a lot of patterns are identified as pattern 2 , and the most state data of SVM classifier have been misjudged. The above results show that the ESMD-RFs model is more effective for axial piston pump fault diagnosis.

5.5. Model Application and Analysis. The remaining 35 sample sets are used as the validation data set of the axial piston pump fault diagnosis model. First, each test sample data set is uploaded to the bucket of OBS on the cloud one by one, and the nodes such as read test set data from OBS, modify metadata, read random forest model, model application, and save data to the data set are used in MLS on the cloud. The validation workflow of the axial piston pump fault diagnosis model is built, as shown in Figure 15.

In the process of fault diagnosis model verification, firstly, according to the fault status of the axial piston pump, the verification data set is divided into 5 categories (each category includes 7 groups of verification data sets), and each category corresponds to a unique label value. Then, 35 sets of 
TABLE 2: Label of axial piston pump state.

\begin{tabular}{lccccc}
\hline Pump status & Normal state & Single slipper wear & Single loose slipper & Swash plate wear & Center spring wear \\
\hline Label & 0 & 1 & 2 & 3 & 4
\end{tabular}

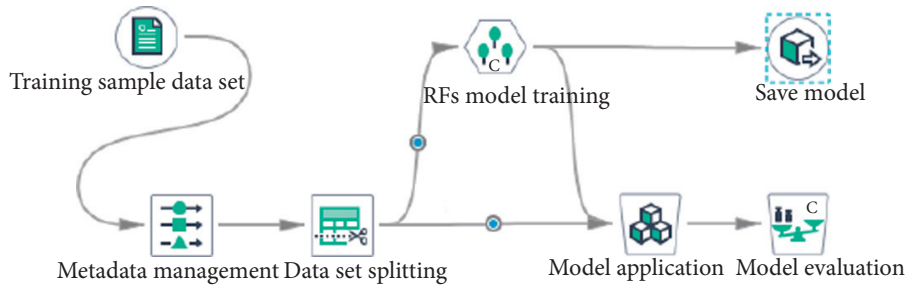

FIGURE 13: Workflow of RFs model training.

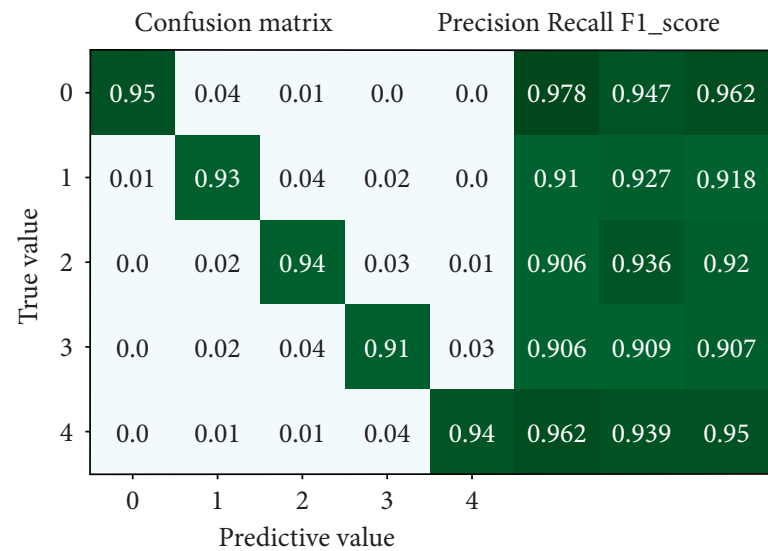

(a)

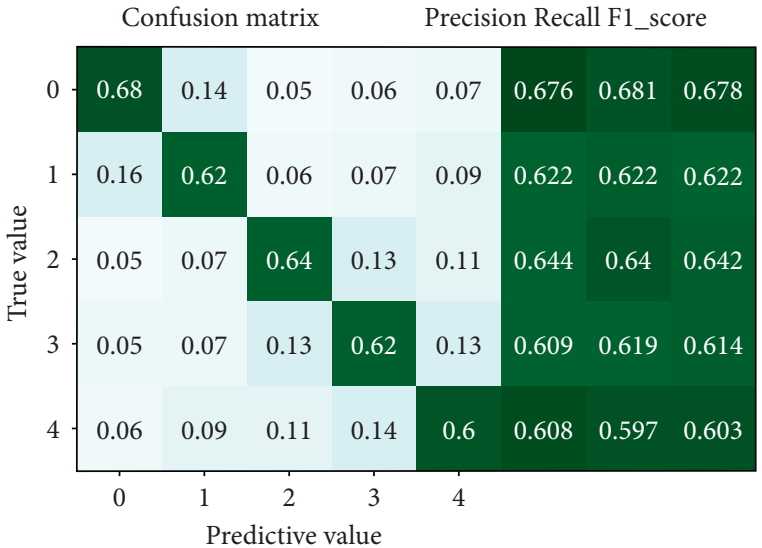

(b)

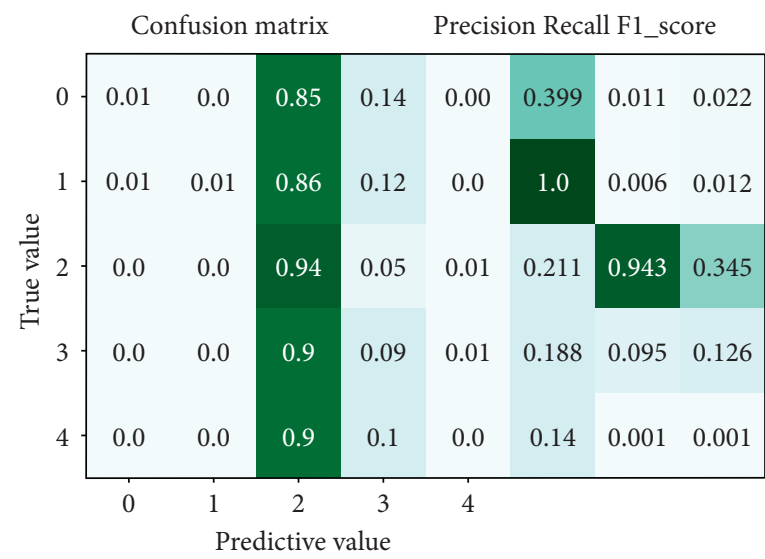

(c)

FIgURE 14: The result of model evaluation. (a) The result of RFs model. (b) The result of CART model. (c) The result of SVM model.

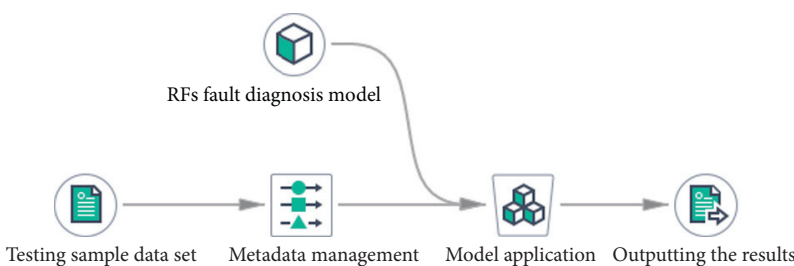

FIGURE 15: Workflow of RFs model testing. the validation set data are inputted into the axial piston pump fault model one by one according to the category, and the model diagnosis output data obtained is shown in Figure 16.

In the process of visual analysis of fault diagnosis data of axial piston pump, the position of the arrow in the radar chart represents the fault diagnosis type of the test set. It can be seen from Figure 16 that for normal state 16(a), single loose slipper 16(c), swash plate wear 16(d), 


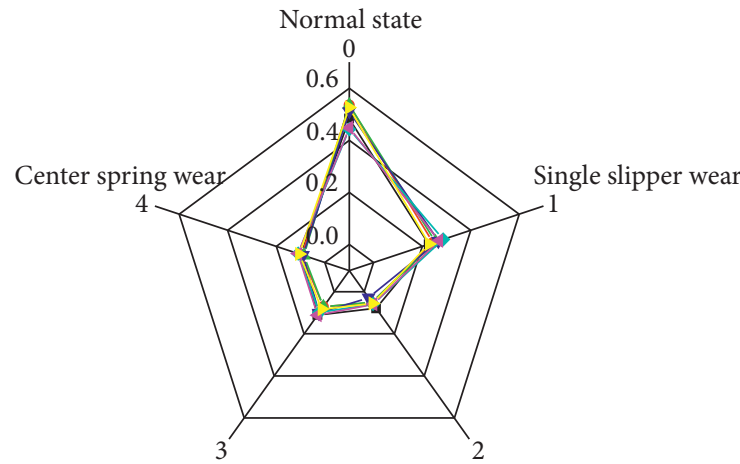

Swash plate wear Single loose slipper

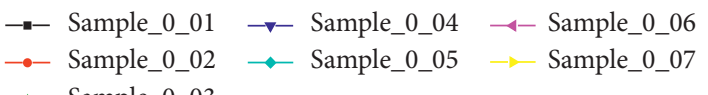

- $\triangle$ - Sample_0_03

(a)

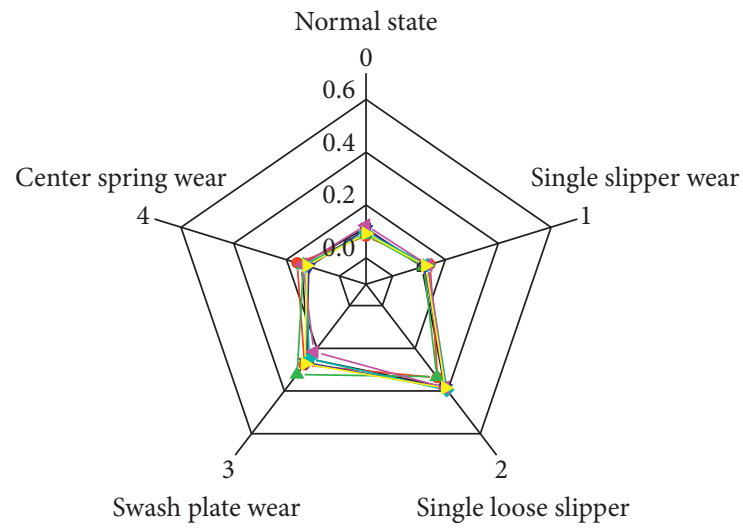

Swash plate wear

$\rightarrow-$ Sample_2_01 $\rightarrow-$ Sample_2_04 $\rightarrow-$ Sample_2_06

$\rightarrow-$ Sample_2_02 $\rightarrow$ Sample_2_05 $\rightarrow-$ Sample_2_07

- - - Sample_2_03

(c)

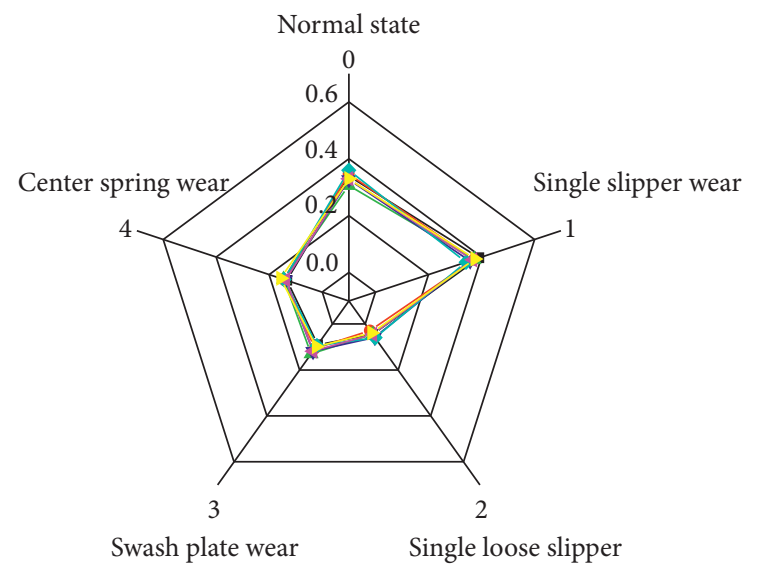

$\rightarrow-$ Sample_1_01 $\rightarrow$ Sample_1_04 $\rightarrow-$ Sample_1_06

$\rightarrow-$ Sample_1_02 $\rightarrow$ Sample_1_05 $\rightarrow$ Sample_1_07

-₫-Sample_1_03

(b)

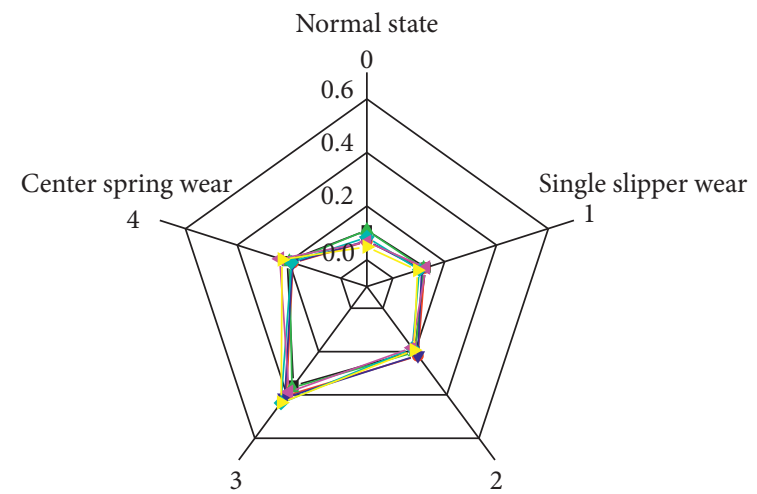

Swash plate wear Single loose slipper

$\rightarrow-$ Sample_3_01 $\rightarrow-$ Sample_3_04 $\rightarrow-$ Sample_3_06
$\rightarrow-$ Sample_3_02 $\rightarrow$ Sample_3_05 $\rightarrow$ Sample_3_07
$\rightarrow-$ Sample_3_03

(d)

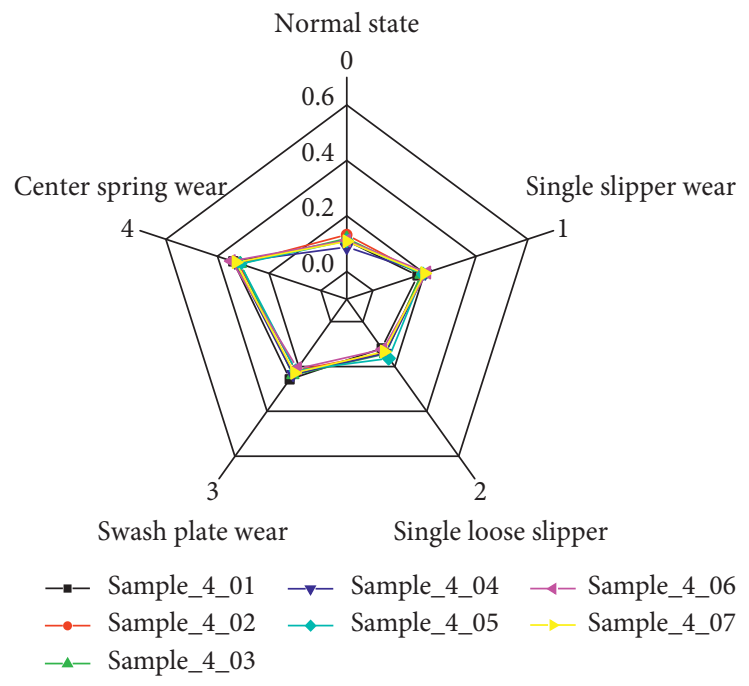

(e)

Figure 16: Test results of fault diagnosis model for axial piston pump. (a) The normal state of fault free. (b) The fault state of single slipper wear. (c) The fault state of single loose slipper. (d) The fault state of swash plate wear. (e) The fault state center spring wear. 
and center spring wear fault 16(e), the four kinds of axial piston pump faults can be depicted clearly. Among them, for the single slipper wear shown in Figure 16(b), there are 6 groups of validation data sets with correct fault diagnosis and 1 group of fault diagnosis error; the fault type is easy to be confused with the normal fault-free state. If the comparison between 16(b) and 16(a) is made, it is found that the fault of the single slipper wear is on the rise, while the normal state is on the decline. This phenomenon reflects that the axial piston pump has had early minor faults and its performance is gradually deteriorating.

In conclusion, the RFs model of axial piston pump fault diagnosis is validated and evaluated with 35 validation data sets. Among them, 34 samples were correctly diagnosed and 1 sample was wrong. The accuracy of this method for axial piston pump fault diagnosis is $97.14 \%$.

\section{Conclusion}

Aiming at the fault diagnosis of axial piston pump, a new remote fault diagnosis method of the axial piston pump is proposed, which combines edge calculation using ESMD and RFs diagnosis algorithm using MLS cloud platform. By testing and verifying the axial piston pump normal fault-free, single slipper wear, single loose slipper wear, swash plate wear, and center spring wear, the precision rates of the model are above $90.6 \%$, the recall rates are more than $90.9 \%$, the F1 score is higher than $90.7 \%$, and the fault diagnosis accuracy of the model is $97.14 \%$, which indicates the accuracy and effectiveness of the method. Besides, the study organically integrates the collection of axial piston pump state monitoring signal, local edge computing feature extraction, and cloud fault diagnosis modeling, which can provide an implementation approach for the construction of axial piston pump remote fault diagnosis system.

\section{Data Availability}

The data can be accessed from the Case Western Reserve University Bearing Data Center Website (http://csegroups. case.edu/bear-ingdatacenter/pages/downloaddata-file). And other .csv data used to support the findings of this study are available from the corresponding author upon request.

\section{Conflicts of Interest}

The authors declare that there are no conflicts of interest regarding the publication of this paper.

\section{Acknowledgments}

This work was funded by the Startup Project of Doctor Scientific Research of North China Institute of Aerospace Engineering (BKY-2021-05), the National Natural Science Foundation of China (51875498), and the Key Program of Hebei Natural Science Foundation (E2018203339).

\section{References}

[1] G. Haidak, D. Wang, and E. L. Ekemeyong Awong, "Modelling of deformation and failure of slipper-retainer assembly in axial piston machine," Engineering Failure Analysis, vol. 111, Article ID 104490, 2020.

[2] Q. Du and K. Zhang, "Condition monitoring and fault diagnosis of hydraulic pump based on inherent vibration signals," Transactions of the Chinese Society of Agricultural Engineering (Transactions of the CSAE), vol. 23, no. 4, pp. 120-123, 2007.

[3] T. Zloto and P. Stryjewski, "Modeling the load of the kinematic pair piston-cylinder in an axial piston pump by means of FEA," Procedia Engineering, vol. 177, pp. 233-240, 2017.

[4] L. Zhou, C. Han, L. Bai, W. Li, M. A. El-Emam, and W. Shi, "CFD-DEM bidirectional coupling simulation and experimental investigation of particle ejections and energy conversion in a spouted bed," Energy, vol. 211, Article ID 118672, 2020.

[5] Y. Yang, L. Zhou, W. Shi, Z. He, Y. Han, and Y. Xiao, "Interstage difference of pressure pulsation in a three-stage electrical submersible pump," Journal of Petroleum Science and Engineering, vol. 196, Article ID 107653, 2020.

[6] G. Peng, X. Huang, L. Zhou, G. Zhou, and H. Zhou, "Solidliquid two-phase flow and wear analysis in a large-scale centrifugal slurry pump," Engineering Failure Analysis, vol. 114, Article ID 104602, 2020.

[7] H. Yan, X. Su, H. Zhang et al., "Design approach and hydrodynamic characteristics of a novel bionic airfoil," Ocean Engineering, vol. 216, Article ID 108076, 2020.

[8] X. Tang, L. Lin, H. Gao, and A. Luo, "Fault feature extraction combining continuous wavelet transform with multiple constraints nonnegative matrix factorization," Journal of Vibration and Shock, vol. 32, no. 19, pp. 7-11, 2013.

[9] R. Zhou, Z. Jiao, and S. Wang, "Current research and developing trends on fault diagnosis of hydraulic systems," Chinese Journal of Mechanical Engineering, vol. 42, no. 9, pp. 6-14, 2006.

[10] N. Helwig, S. Klein, and A. Schütze, "Identification and quantification of hydraulic system faults based on multivariate statistics using spectral vibration features," Procedia Engineering, vol. 120, no. 1, pp. 1225-1228, 2015.

[11] C. Qin, G. Shi, J. Tao et al., "Precise cutterhead torque prediction for shield tunneling machines using a novel hybrid deep neural network," Mechanical Systems and Signal Processing, vol. 151, Article ID 107386, 2021.

[12] C. Qin, J. Tao, H. Shi, D. Xiao, B. Li, and C. Liu, "A novel Chebyshev-wavelet-based approach for accurate and fast prediction of milling stability," Precision Engineering, vol. 62, pp. 244-255, 2020.

[13] Y. Jin, C. Qin, Y. Huang, and C. Liu, "Actual bearing compound fault diagnosis based on active learning and decoupling attentional residual network," Measurement, vol. 173, Article ID 108500, 2021.

[14] P. Casoli, M. Pastori, F. Scolari, and M. Rundo, “A vibration signal-based method for fault identification and classification in hydraulic axial piston pumps," Energies, vol. 12, no. 5, p. 953, 2019.

[15] S. Li, P. Zhang, B. Li, and G. Wang, "Fault feature selection method for axial piston pump based on quantum genetic algorithm," China Mechanical Engineering, vol. 25, no. 12, pp. 1659-1644, 2014.

[16] S. Liu, Y. He, X. Li, M. Lu, and Z. Lu, "Multi-information intensity characteristic state assessment method of hydraulic 
pumps based on fault mechanism," China Mechanical Engineering, vol. 30, no. 12, pp. 1460-1465, 2019.

[17] P. Wang, X. Wang, H. Zhu, Y. Li, and M. Zhang, "A fault diagnosis method based on sparse coding for hydraulic pump," Transactions of Beijing Institute of Technology, vol. 37, no. 5, pp. 451-454, 2017.

[18] D. Buono, D. Siano, E. Frosina, and A. Senatore, "Gerotor pump cavitation monitoring and fault diagnosis using vibration analysis through the employment of auto-regressivemoving-average technique," Simulation Modelling Practice and Theory, vol. 71, pp. 61-82, 2017.

[19] Y. Jia, M. Xu, and R. Wang, "Symbolic important point perceptually and hidden markov model based hydraulic pump fault diagnosis method," Sensors, vol. 18, no. 12, p. 4460, 2018.

[20] S. Liu, L. Zhang, X. Zeng, and Y. Lan, "Loose slipper fault diagnosis of axial piston pump based on MED-EEMD and ELM," Journal of Mechanical\& Electrical Engineering, vol. 37, no. 3, pp. 241-246, 2020.

[21] C. Lu, S. Wang, and C. Zhang, "Fault diagnosis of hydraulic piston pumps based on a two-step EMD method and fuzzy Cmeans clustering," Proceedings of the Institution of Mechanical Engineers, Part C: Journal of Mechanical Engineering Science, vol. 230, no. 16, pp. 2913-2928, 2016.

[22] Y. Tian, C. Lu, Z. Wang, and Z. Wang, "Fault diagnosis based on LMD-SVD and information-geometric support vector machine for hydraulic pumps," Transactions of the Canadian Society for Mechanical Engineering, vol. 39, no. 3, pp. 569-580, 2015.

[23] Z. Zheng, W. Jiang, B. Wang, and Y. Wang, "Hydraulic pump fault diagnosis method of symplectic geometry mode decomposition and generalized morphological fractal dimensions," Journal of Harbin Engineering University, vol. 41, no. 5, pp. 724-730, 2020.

[24] Z. Du, J. Zhao, and X. Zhang, "Research on fault diagnosis method of hydraulic pump based on sensitivity analysis and PNN," Machine Tool \& Hydraulics, vol. 46, no. 19, pp. 171175, 2018.

[25] H. A. Babikir, M. A. Elaziz, A. H. Elsheikh et al., "Noise prediction of axial piston pump based on different valve materials using a modified artificial neural network model," Alexandria Engineering Journal, vol. 58, no. 3, pp. 1077-1087, 2019.

[26] Y. Lv, L. Fang, and Q. Zhang, "Application of LCD fuzzy entropy and SOM neural network in fault diagnosis of hydraulic pump," Machine Tool \& Hydraulics, vol. 44, no. 9, pp. 178-182, 2016.

[27] J. G. M. Lázaro and C. B. Pinilla, "A methodology for detection of wear in hydraulic axial piston pumps," International Journal on Interactive Design and Manufacturing (IJIDeM), vol. 14, no. 3, pp. 1103-1119, 2020.

[28] M. Shen, L. Zhao, B. Zhou, and C. Zhou, "Hydraulic pump fault diagnosis of RBF network based on PSO optimization," Chinese Hydraulic \& Pneumatics, vol. 5, pp. 87-92, 2016.

[29] D. Bensaad, A. Soualhi, and F. Guillet, "A new leaky piston identification method in an axial piston pump based on the extended Kalman filter," Measurement, vol. 148, Article ID 106921, 2019.

[30] W. Cai, K. Huang, M. Dai, and Z. Yang, "Fault diagnosis of piston pumps based on wavelet packet decomposition and DAG SVM," Chinese Hydraulic \& Pneumatics, vol. 12, pp. 12-15, 2015.

[31] K. Han, W. Jiang, Y. Lei, Y. Zhang, and S. Zhang, "Performance degradation assessment method of hydraulic pump based on integrated VMD and SVDD," Machine Tool \& Hydraulics, vol. 47, no. 19, pp. 164-170, 2019.

[32] J. Wang and Z. Li, "Extreme-point symmetric mode decomposition method for data analysis," Advances in Adaptive Data Analysis, vol. 5, no. 3, pp. 1137-1148, 2013.

[33] W. Li, Z. Tian, W. Gong, and W. Wang, "Developed ESMD for the feature extraction of abnormal sound in public places," Chinese Journal of Scientific Instrument, vol. 37, no. 11, pp. 2429-2437, 2016.

[34] W.-A. Yang, W. Zhou, W. Liao, and Y. Guo, "Identification and quantification of concurrent control chart patterns using extreme-point symmetric mode decomposition and extreme learning machines," Neurocomputing, vol. 147, pp. 260-270, 2015.

[35] S. Zhang, J. Xu, A. Jiang, J. Li, X. Su, and W. Jiang, "Fault diagnosis of bearings based on extreme point symmetric mode decomposition and probabilistic neural network," China Mechanical Engineering, vol. 28, no. 4, pp. 425-431, 2017.

[36] L. Breiman, "Random forests," Machine Learning, vol. 45, no. 1, pp. 5-32, 2001.

[37] J. Liu, M. Fu, and J. Tang, "MFL inner detection based defect recognition method," Chinese Journal of Scientific Instrument, vol. 37, no. 11, pp. 2572-2581, 2016.

[38] M. Cerrada, G. Zurita, D. Cabrera, R.-V. Sánchez, M. Artés, and $\mathrm{C} . \mathrm{Li}$, "Fault diagnosis in spur gears based on genetic algorithm and random forest," Mechanical Systems and Signal Processing, vol. 70-71, pp. 87-103, 2016.

[39] J. Wang, B. Xiong, Y. Mo, J. Huang, X. Li, and P. Zhao, "Recognition method of helicopter flight condition based on random forest," Computer Engineering and Applications, vol. 53, no. 17, pp. 149-152, 2017.

[40] Y. Lei, F. Jia, D. Kong, J. Lin, and S. Xing, "Opportunities and challenges of machinery intelligent fault diagnosis in big data era," Journal of Mechanical Engineering, vol. 54, no. 5, pp. 94-104, 2018.

[41] D. Michie, D. J. Spiegelhalter, and C. C. Taylor, "Machine learning," Neural and Statistical Classification, vol. 13, pp. 1-298, 1994.

[42] H. Lü, D. Chen, B. Fan, Y. Wang, and Y. Wu, "Standardization progress and case analysis of edge computing," Journal of Computer Research and Development, vol. 55, no. 3, pp. 487-511, 2018.

[43] W. Shi, H. Sun, J. Cao, Q. Zhang, and W. Liu, "Edge computing-an emerging computing model for the internet of everything era," Journal of Computer Research and Development, vol. 54, no. 5, pp. 907-924, 2017.

[44] J. Yue, G. Yang, and H. Feng, "Comparative of remote sensing estimation models of winter wheat biomass based on random forest algorithm," Transactions of the Chinese Society of Agricultural Engineering (Transactions of the CSAE), vol. 32, no. 18, pp. 175-182, 2016. 